\title{
O Módulo Raciocinador do Sistema de Avaliação da Aprendizagem (SAA) aplicado ao Ensino de Algoritmos: um potencializador de cursos nas modalidades EaD, Convencional e Híbrida.
}

\author{
Dilermando Piva Jr. ${ }^{1}$; Francisco de Assis de Freitas ${ }^{1}$; \\ ${ }^{1}$ Centro Paula Souza - Faculdade de Tecnologia de Itu, SP. \\ Av. Tiradentes, 1.211, Itu, SP \\ pivajr@gmail.com, freitas.assis@gmail.com
}

\begin{abstract}
Resumo. Este trabalho apresenta o sistema SAA, em específico o módulo raciocinador, que foi especificado para auxiliar professores na redefinição de conteúdos para alunos que apresentam dificuldades na assimilação dos tópicos explorados. Para isso, existe um mecanismo que, além de facilitar o processo de avaliação, procura por casos similares ocorridos com outros alunos em situações passadas semelhantes. Esta procura permite que o sistema possa redefinir o conteúdo a que o aluno deve ser submetido, para eliminar as deficiências detectadas durante a avaliação formativa, sem a necessidade de uma maior intervenção do professor. Para atingir tal objetivo, são explorados conceitos de Inteligência Artificial (especificamente Raciocínio Baseado em Casos). Estudos preliminares apontam uma efetividade de redirecionamento superior a $50 \%$.
\end{abstract}

Palavras Chave: Ensino Online, RBC, Avaliação Formativa, SAA.

\begin{abstract}
This work presents the SAA system, specifically the reasoning module, which was specified to help teachers in the redefinition of content for students who present difficulties in assimilating the topics explored. To this end, there exists a mechanism that ease the evaluation process and search for similar cases involving other students in past situations. This search enables the system to redefine the content of the information to which the student will be presented, so that deficiencies detected during his/her formative evaluation can be eliminated without a considerable intervention of the teacher. To achieve this goal, concepts of Artificial Intelligence (Case-Based Reasoning, more specifically) are explored. Preliminary studies indicate a redirection effectiveness of more than $50 \%$.
\end{abstract}

Keywords: Online Teaching, CBR, Formative Assessment, SAA.

\section{Introdução}

Muito se tem escrito sobre métodos, metodologias, ferramentas, ambientes, práticas e técnicas adotadas no ensino de Algoritmos. Uma revisão dos últimos trabalhos publicados nesta área nos remete a uma análise de, fundamentalmente, três perspectivas: Utilização e/ou construção de ferramentas específicas para o ensino/aprendizagem de algoritmos, com destaque para Cares (2002), Alice (2015), Azul, Mendes (2015); 
VI Congresso Brasileiro de Informática na Educação (CBIE 2017)

Anais dos Workshops do VI Congresso Brasileiro de Informática na Educação (WCBIE 2017)

Dellazzana (2015) e Scratch (2015); Estratégias de ensino/aprendizagem que utilizam ambientes lúdicos e jogos educacionais para o ensino de algoritmos (Borges 2002; Rodrigues 2004; Tarouco, 2005; Priesnitz Filho, Abegg, Simonetto 2012, dentre outros); e Utilização e/ou construção de ambientes Web para ensino e/ou auxilio da disciplina de algoritmos, com os trabalhos de Pereira Jr., Rapkiwicz (2004), Delgado, Xexeo, Souza, Rapkiewicz, Pereira Jr. (2005) e Black 2015, dentre muitos outros.

Além dessas modalidades e ambientes analisados e indicados, existem outros criados para auxiliar estudantes e professores no ensino de algoritmos e linguagem de programação. Os autores Camila et. al (Santos et. al 2014) analisaram nove ferramentas utilizadas para o ensino de algoritmos. Delas, 77\% apresentam suporte para a representação de algoritmos por meio de fluxograma ou portugol. Além disso, $66 \%$ possibilitam a execução passo-a-passo, o que facilita a visualização da execução e as alterações de um passo (comando) para o outro. Oitenta e oito por cento dessas ferramentas $(88 \%)$ não apresentam suporte para o ambiente Web.

Entretanto, nos trabalhos existentes, não foram observadas funcionalidades nas ferramentas que auxiliassem os docentes na identificação explicita das deficiências no processo de aprendizagem de algoritmos e linguagens de programação ou, tampouco, funcionalidades que indicassem ações que os estudantes devessem tomar para melhorar determinados conceitos. Mesmo assim, é certo que ambientes como estes, podem e devem ser utilizados pelos docentes para indicar alternativas de aprendizagem aos estudantes, conforme metodologia adotada nas respectivas disciplinas.

Foi essa ausência de informações mais voltadas ao gerenciamento do processo de aprendizagem dos estudantes que nos motivou a procurar alternativas que pudessem potencializar a ação docente, surgindo assim o SAA (Piva Jr et al, 2016).

Por outro lado, é crescente o número de instituições de ensino que utilizam o canal web (ensino online) para oferecimento de cursos, formais ou não. Isto vem motivando Universidades de todo o mundo a "repensarem" suas práticas e políticas educacionais e implantarem não só programas especificamente de educação a distância, mas também potencializarem seus cursos "tradicionais" com modalidades híbridas.

Entretanto, o nível de utilização do ensino online, mesmo que híbrido, está longe de atingir sua máxima potencialidade. Uma série de estudos e pesquisas realizadas ao redor do mundo apontam diversos obstáculos percebidos pelos professores, de ordem pessoal, tecnológica e institucional, como sendo causa de tal limitação.

A partir dessas observações e identificando o potencial da tecnologia para auxiliar o docente na condução de suas disciplinas, provendo ferramental para liberá-lo de tarefas repetitivas, e potencializar o processo de apontamento de deficiências dos estudantes no processo de aprendizagem, foi agregado ao SAA uma funcionalidade de proatividade de indicação do que deve ser estudado para reforço de deficiências percebidas ao longo do processo de aprendizagem do estudante. De forma simples, o módulo raciocinador controla o caminho percorrido pelo aluno ao logo do conteúdo explorado no curso, sendo responsável por redefinir, de forma automática, o conteúdo onde o aluno apresentou deficiências, de acordo com o caminho percorrido e as avaliações que foram realizadas. Para isso, utiliza a abordagem de Inteligência Artificial conhecida como Raciocínio Baseado em Casos (RBC) (Kolodner, 1993). Assim, este artigo tem como objetivo apresentar esse novo módulo do sistema SAA, mostrando suas características, arquitetura e forma de funcionamento. 
VI Congresso Brasileiro de Informática na Educação (CBIE 2017)

Anais dos Workshops do VI Congresso Brasileiro de Informática na Educação (WCBIE 2017)

\section{Obstáculos para a Introdução do Ensino Online}

Para conseguir sucesso na integração da tecnologia ao ensino, não devemos apenas nos ater à aceitação e efetiva utilização da tecnologia pelos estudantes, mas também, e de forma prioritária, do seu entendimento e sua massiva utilização por parte dos professores. No caso do ensino online, e suas variantes, estudos relatam uma série de obstáculos (barreiras) que impedem o efetivo uso da tecnologia pelos professores, fazendo surgir um hiato entre o uso atual e o esperado.

Já em 2001, Pajo e Wallace (2001) baseados no resultado de uma pesquisa realizada junto aos professores da Universidade Massey (Nova Zelândia), apontam vários obstáculos, dentre eles: o tempo necessário para usar os ambientes de ensino online e monitoramento do curso (feedback aos alunos). Resultados semelhantes podem ser encontrados em (Metcalf, 1997), (Hare; McCartan, 1996), (Grace; Smith 2001), entre outros. Revisões da literatura e artigos mais recentes apontam resultados semelhantes, porém mais adequados a nossa realidade tecnológica. São exemplos: (Maguire, 2008), (Guri-Rosenblit, 2005), (Clark, 2009) e (Muilenburg; Berge, 2009).

A partir dessas constatações, identificamos uma das barreiras recorrentes, não só para consecução dos cursos na modalidade a distância, mas também nas classes presenciais ou hibridas: o tempo necessário para monitoramento do curso (entendimento do rendimento e feedback aos estudantes). Assim, o módulo raciocinador do sistema SAA, concentra esforços para reduzir esse obstáculo, em específico.

\section{O Modelo de Ensino Proposto}

Através dos estudos realizados, foi possível verificar que além da reconstrução e formatação dos conteúdos, utilizando técnicas inerentes à internet e ao processo de ensino a distância (tais como hipertexto, linguagem dialógica, semiótica e conceitos de interação homem-máquina), a avaliação do aprendizado destes conteúdos mostra-se de grande importância. Entende-se que, dentro de um curso oferecido à distância, cada conceito tem a sua própria forma de avaliação e exposição de materiais instrucionais.

A prática didática no ensino superior mostra que a ideia de conceito pode ser representada, a grosso modo, pela noção de uma aula. Dessa forma, os conteúdos foram agregados por aula, e a cada aula associado um conceito (tópico da matéria) a ser ensinado. A esse conceito ou aula, foram associadas uma série de informações, inclusive como esse determinado conceito deve ser avaliado e tido como aprendido. Nesse sentido são associados a ele, um conjunto de questões, com vários níveis de dificuldade, e então guardados em uma base de questões. A Figura 1, a seguir, representa a sequência de planejamento de uma determinada disciplina que deve ser realizada por um docente no sistema SAA. Uma vez realizados os cadastros iniciais, deve-se observar as etapas operacionais frequentes do sistema. Em termos práticos, uma vez que se chega a uma data planejada de aula, o sistema aponta que pode ser realizado o lançamento das presenças dos estudantes.

Uma vez apontadas as presenças, apenas e tão somente para os alunos que estiveram presentes a aquela atividade, o sistema irá montar uma avaliação (individualizada) e será associada aos estudantes que estiveram presentes. Uma mensagem por e-mail será enviada a cada estudante presente para que ele se lembre de que existe uma avaliação a ser realizada, com informações da disciplina, da aula e a quantidade de minutos que o estudante deve reservar (em média) para respondê-la. 


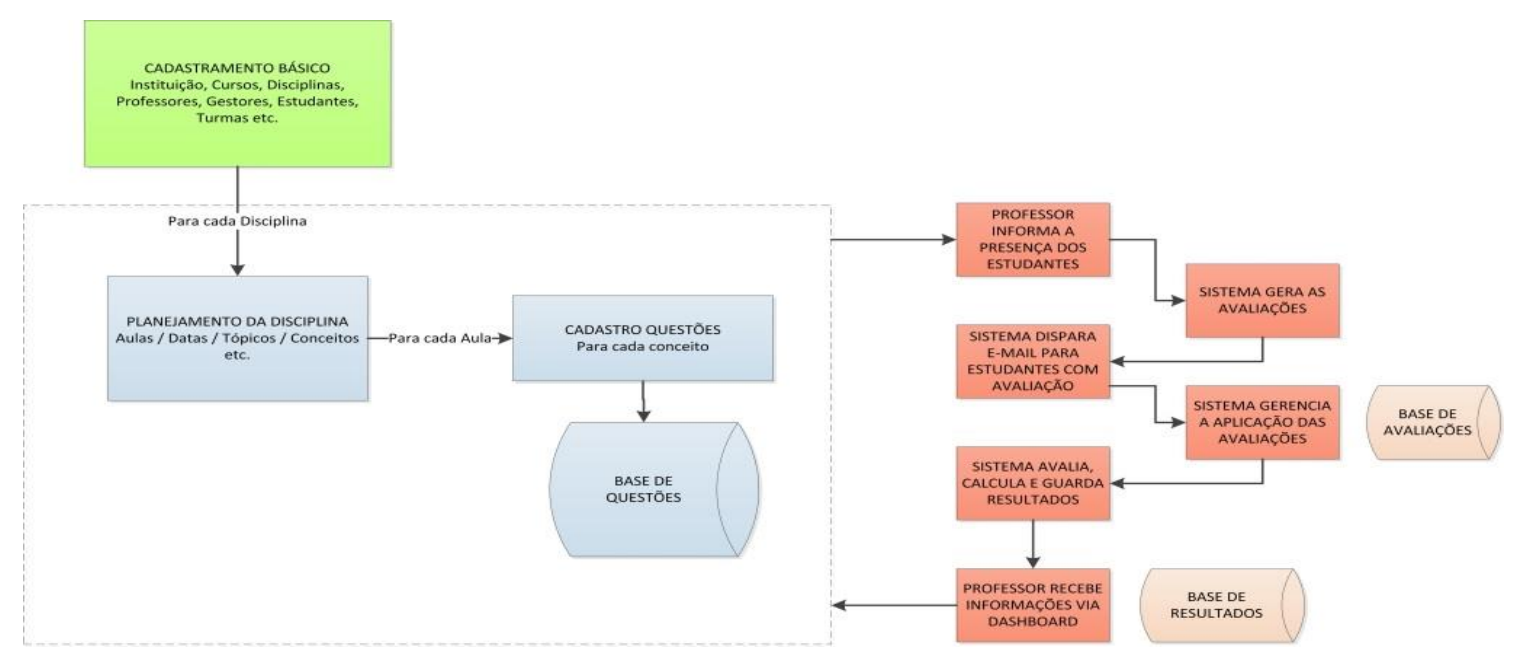

Figura 1 - Etapas iniciais de cadastramento e operacionais no sistema SAA

Uma vez respondida uma avaliação, o sistema disponibilizará uma série de relatórios e gráficos para que o professor, o estudante, o coordenador e o diretor da unidade, possam tomar as ações convenientes.

Até aqui, as ações tomadas, principalmente pelos estudantes, estavam baseadas em suas próprias percepções de seu processo de aprendizado. Com a implementação do módulo raciocinador, o sistema SAA, passa a apresentar sugestões aos estudantes para que eles possam melhor perceber suas deficiências e o que pode ser feito para transpôlas.

Isso é possível pois, os conteúdos são armazenados em Bases de Conhecimento, o que facilita a sua manutenção e posterior recuperação. Para a recuperação dessas informações, baseada no perfil do aluno, são utilizadas técnicas de Inteligência Artificial, especificamente RBC. Isto facilita a questão de migração simétrica entre os conceitos (aulas), dado um problema de aprendizagem.

Cabe ressaltar que, na montagem automática da avaliação pelo sistema, o professor deverá fornecer o Critério mais adequado de Avaliação daquele determinado conteúdo. Existindo compatibilidade entre a base de questões e o critério de avaliação, o sistema montará, automaticamente a Avaliação.

\section{O Módulo Raciocinador do Sistema SAA}

A Figura 2 ilustra de forma simplificada o funcionamento do módulo raciocinador do sistema SAA. Os alunos iniciam o Módulo de Instrução no conceito 1 (ou Aula 1). Após o desenvolvimento deste conceito, aplica-se uma avaliação (formativa) dos pontos centrais de tal conceito. Esta avaliação é recuperada da base de questões seguindo os critérios de validação inicialmente introduzidos pelo professor na montagem do curso.

Neste processo de passagem por cada um dos conteúdos e avaliações, o sistema SAA fará o registro, montando assim o Modelo do Aluno pelo caminho de aprendizagem, suas capacidades (exercícios resolvidos acertadamente), as atitudes (exercícios resolvidos utilizando adaptações ou outro tipo de ajuda solicitada pelo aluno) e suas limitações (exercícios que o aluno encontrou dificuldades). Estes atributos comporão o perfil do aluno (tais informações serão armazenadas no modelo do aluno), 
VI Congresso Brasileiro de Informática na Educação (CBIE 2017)

Anais dos Workshops do VI Congresso Brasileiro de Informática na Educação (WCBIE 2017)

que será utilizado na situação em que os módulos de instrução não forem suficientes para a consecução da aprendizagem de um determinado conceito, sendo então remetido aos módulos TD (Tira Dúvida) e DC (Determina Conteúdo) do sistema SAA

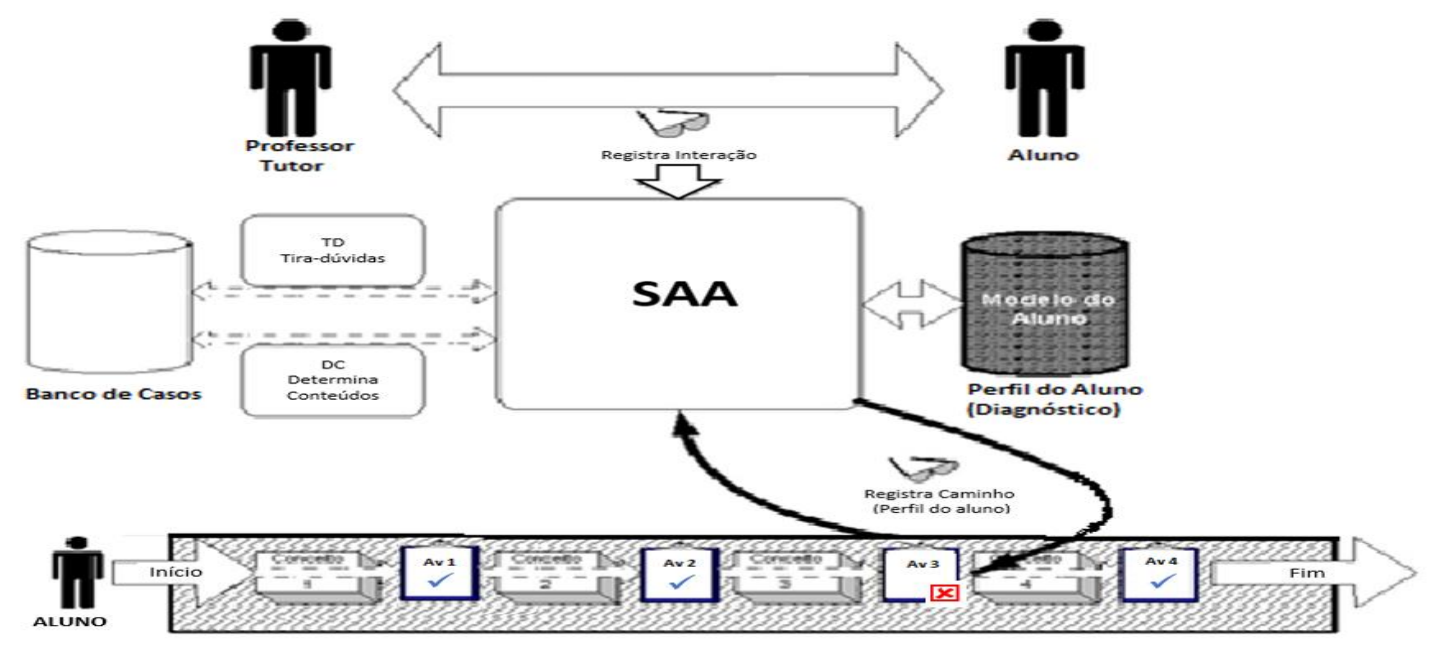

Figura 2: Mini-Arquitetura do Módulo Raciocinador do sistema SAA: Relacionamento entre o Curso, a Biblioteca de Casos e o Professor.

Estes, por sua vez, procurarão na Base de Casos (Base de Conhecimento) do sistema, um caso que se enquadre no perfil do aluno. Caso exista, este lhe será exibido. Caso contrário, uma intervenção do professor será solicitada. Essas intervenções do professor serão totalmente registradas para fins de montagem de novos Casos Pedagógicos específicos para o correspondente perfil de aluno, sendo, por fim, armazenadas na Base de Casos do Sistema SAA. Após esta intervenção, o conceito é dado como compreendido.

\subsection{O Modelo do Aluno}

Existem muitas técnicas para a implementação do Modelo do Aluno na literatura, porém a maior parte destas técnicas é computacionalmente complexa. Como é o caso das técnicas numéricas: Redes Bayesianas, ou a teoria da evidência de Dempster-Shafer, ou ainda a modelagem nebulosa ("fuzzy") do aluno. Outras técnicas, embora computacionalmente mais simples, (por exemplo, a modelagem pelo traçado de aproximação), ficam restritas ao registro do que o aluno sabe, mas não reflete o seu comportamento e características.

No sistema SAA o Modelo do Aluno armazena as informações do percurso do estudante durante o processo de aprendizagem, caracterizando assim, o seu perfil. Como descrito anteriormente, para o gerenciamento dessas informações, utiliza-se a abordagem de RBC. Isto permite que dada a necessidade de intervenção do professor no processo de aprendizado de um dado aluno, exista a possibilidade de associação entre a intervenção e o perfil do aluno. A partir desta situação (Caso), consegue-se o registro da mesma na Base de Casos do sistema (na forma de um Caso), o qual, após seu registro, ficará disponível para uma possível utilização futura, evitando a repetição da intervenção do professor. Portanto, a modelagem de tal técnica é simples e não requer computacionalmente a inferência de complexos algoritmos. 
VI Congresso Brasileiro de Informática na Educação (CBIE 2017)

Anais dos Workshops do VI Congresso Brasileiro de Informática na Educação (WCBIE 2017)

\subsection{Elementos do Modelo do Aluno}

O Modelo do Aluno representa o conhecimento sobre o estudante, permitindo com tais informações que um Sistema Baseado em Casos as utilize para prover instruções (ou feedback) adaptáveis àquele estudante (possibilitando a individualização do processo de aprendizagem e redução da necessidade de intervenções do docente). Dessa forma, o Modelo do Aluno, adaptado ao modelo Problema-Solução, utilizado em RBC, possibilita que se tenha um Caso sendo representado conforme ilustrado na Tabela 1.

O Problema (sintomas) é composto pelo conjunto de atributos que definem os índices para um futuro reaproveitamento da Solução, onde estará o material que fornecerá de forma automática o aprofundamento de conteúdo exigido pelo aluno. Os Casos serão estruturados em uma Base de Casos que apresenta uma organização hierárquica - Figura 3.

Tabela 1: Estrutura de um Caso, adaptado ao Modelo do Aluno

\begin{tabular}{|c|c|}
\hline $\begin{array}{l}\text { C } \\
\text { A } \\
\text { S } \\
0 \\
n\end{array}$ & 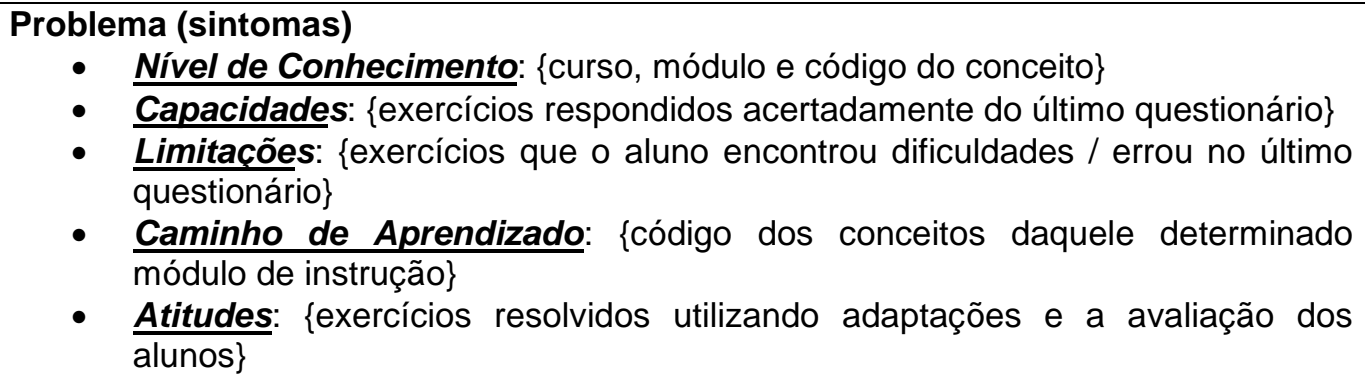 \\
\hline & $\begin{array}{l}\text { Solução } \\
\qquad \begin{array}{l}\text { Intervenção Professor: } \text { \{Descrição, em forma de texto, do que e como } \\
\text { estudar, com referências a links contendo vídeos e outros materiais didáticos. }\end{array}\end{array}$ \\
\hline
\end{tabular}

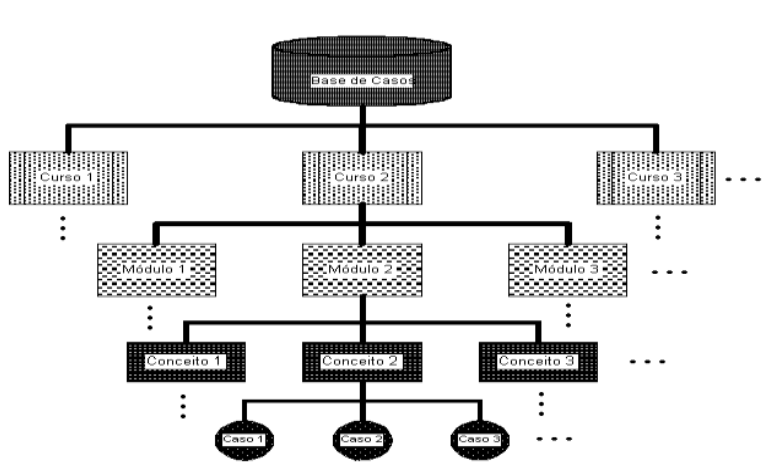

Figura 3: Organização Hierárquica da Base de Casos do Sistema SAA

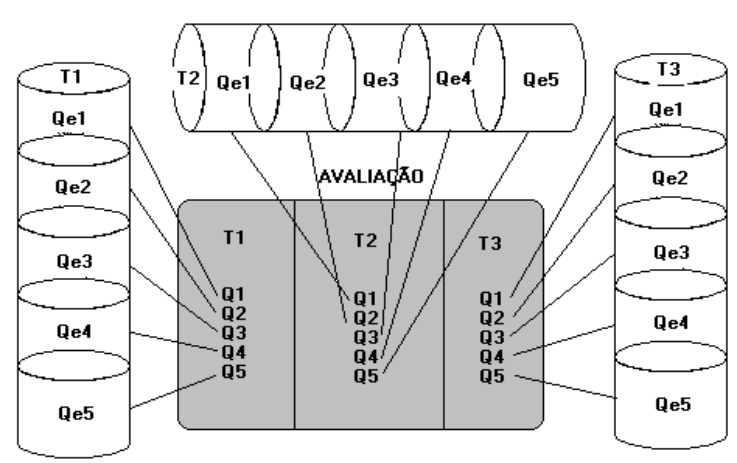

Figura 4 - Processo de Composição da Avaliação no Sistema SAA

Esta organização hierárquica procura dividir os casos em grupos. No nível mais alto da hierarquia tem-se o curso, onde é caracterizado o nome do curso em questão. No primeiro nível intermediário têm-se os diferentes módulos (também podem ser as disciplinas) que compõem um curso. No nível intermediário seguinte, têm-se os vários conceitos (ou aulas) que fazem parte de cada módulo (disciplina) e, por fim, no nível mais baixo tem-se os Casos propriamente ditos, que são agrupados em função dos conceitos. A finalidade desta organização hierárquica reside na preocupação em reduzir 
VI Congresso Brasileiro de Informática na Educação (CBIE 2017)

Anais dos Workshops do VI Congresso Brasileiro de Informática na Educação (WCBIE 2017)

o espaço de análise dos casos potencialmente similares dentro da Base de Casos. Assim, o sistema irá concentrar seu esforço de análise apenas nos casos com real potencial de reaproveitamento.

\subsection{O processo de recuperação dos casos - Cálculo da Similaridade}

O processo de recuperação de um determinado caso na Base de Casos acontecerá em três etapas. A primeira delas é a determinação do nível de conhecimento do aluno, ou seja, acontecerá a delimitação daqueles casos que satisfaçam aos atributos restritivos (Curso + Módulo + Conceito/Aula). Por exemplo na Figura 3, supondo Curso $=2$, Módulo $=2$ e Conceito/Aula $=2$, têm-se como casos pré-selecionados: Caso1, Caso2, Caso3,...CasoN, que compõem o segmento da Base de Casos a ser investigado.

Uma vez identificado o segmento da Base de Casos, verificam-se os casos que estejam no âmbito da confluência de outros dois atributos: Limitações e Capacidades. Como Limitações tem-se a lista de exercícios que o aluno errou durante a avaliação. Já como Capacidades tem-se a lista de exercícios que o aluno acertou na mesma avaliação. Para melhor compreender a avaliação observe a Figura 4. Nela, observa-se que a avaliação é composta de 3 conjuntos (T1,T2 e T3) contendo 5 questões cada. Para compor automaticamente um conjunto, o sistema irá sortear as 5 questões de 5 grupos de questões equivalentes (Qe1-Qe5), ou seja, deverão ser cadastradas várias questões para cada Qe, de onde o sistema irá sortear uma de cada grupo de equivalência. As questões T1 são do tipo "fáceis". Já as questões T2 serão, conceitualmente, mais difíceis do que T1, ficando no nível de dificuldade Médio. Finalmente as questões T3 serão as mais difíceis, estando próximo dos níveis mais elevados da Taxionomia de Bloom. Os pesos para T1, T2 e T3 são configuráveis pelo professor. Como exemplo, tem-se para Capacidades uma Lista $=\{\mathrm{T} 1 \mathrm{Qe} 1, \mathrm{~T} 1 \mathrm{Qe} 3, \mathrm{~T} 2 \mathrm{Qe} 4\}$, indicando que o aluno acertou as questões dos grupos de equivalência 1 e 3 para o conjunto T1 e a questão do grupo 4 para o conjunto T2. A lista para Limitações é composta da mesma forma, porém com os exercícios que o aluno errou.

Tal associação entre as Capacidades e as Limitações indicará uma determinada área, na qual pode ou não existir casos. A Figura 5 ilustra tal definição. Na existência de mais de um caso nesta determinada área, existirá a necessidade de análise dos mesmos para verificação do mais apropriado para recuperação. Neste caso, utilizar-se-á a técnica "do vizinho mais próximo" para recuperação do caso. Segundo Watson (1997), "a técnica do vizinho mais próximo é talvez a mais usada para o estabelecimento da similaridade já que a maioria das ferramentas disponível a utiliza".

Nesta técnica, a similaridade entre o caso de entrada e um caso na Base de Casos é determinada para cada atributo. Esta medida deve ser multiplicada por um fator peso. A somatória de todos os atributos é calculada e permite estabelecer a média de similaridade entre os casos da Base e os de entrada. Nesta etapa os atributos a serem utilizados serão: Caminho de Aprendizado e Atitudes. A formula também é ilustrada na Figura 5.

O resultado de tal operação será uma lista de casos, com seus respectivos valores de similaridade com o caso de entrada. Dessa forma, será recuperado o caso com a maior pontuação, indicando estar mais próximo do caso de entrada, consequentemente maior é a sua similaridade. 
VI Congresso Brasileiro de Informática na Educação (CBIE 2017)

Anais dos Workshops do VI Congresso Brasileiro de Informática na Educação (WCBIE 2017)
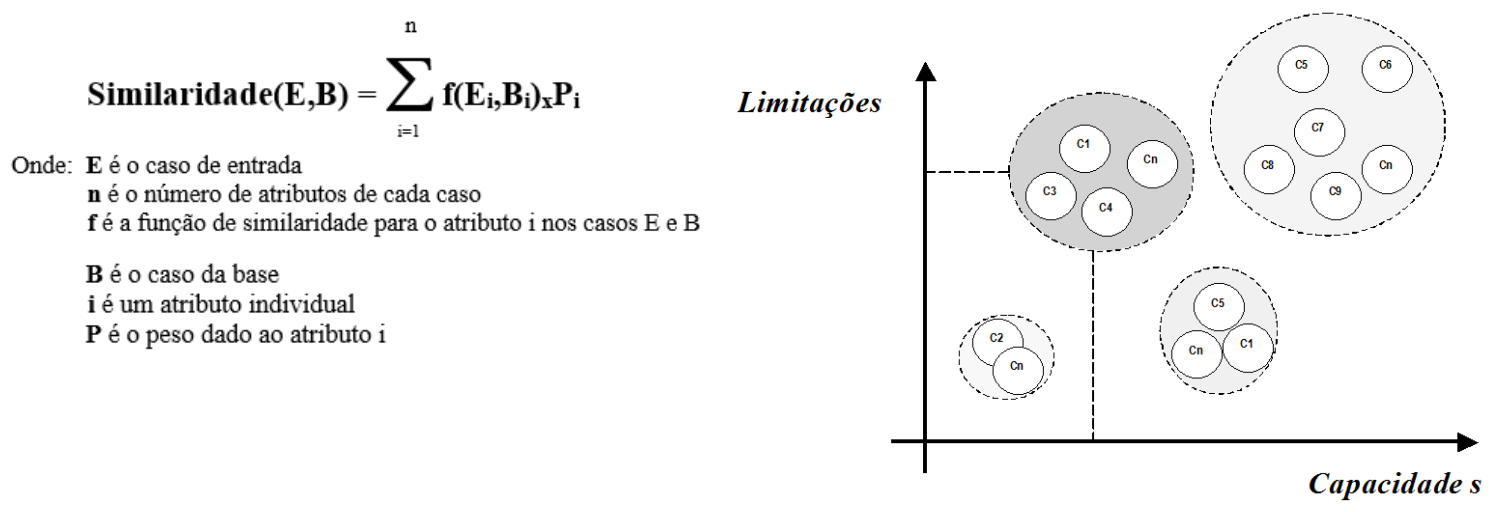

Figura 5 - Processo de Recuperação de Casos no Sistema SAA

Um dos pontos nevrálgicos da utilização da técnica do "vizinho mais próximo" em RBC para a recuperação de informações, consiste no aumento da base de casos. Segundo Watson (1997), a latência de recuperação de casos aumenta de forma linear ao aumento da quantidade de casos. Entretanto, isto não é evidenciado no presente sistema, pois como existem atributos restritivos (Nível de Conhecimento), isto reduz substancialmente o número de casos a serem analisados, possibilitando uma boa performance do sistema, mesmo com uma grande quantidade de casos armazenados.

No final do processo de recuperação, o estudante avalia se o caso foi ou não útil. Essa informação retroalimentará o indicador de Atitudes, potencializando ou não um determinado caso, para um determinado perfil de Aluno.

\section{Considerações Finais}

Este trabalho apresenta uma visão geral do módulo raciocinador do Sistema SAA. Testes preliminares apontam para um percentual médio de recuperação de casos na ordem de 75,24\%. Desses casos recuperados, a média de efetividade foi de $25,43 \%$. A Figura 6 ilustra esse processo testado por 16 dias em três turmas da disciplina de Algoritmos, em duas Faculdades distintas, em duas aulas (conceitos) diferentes.

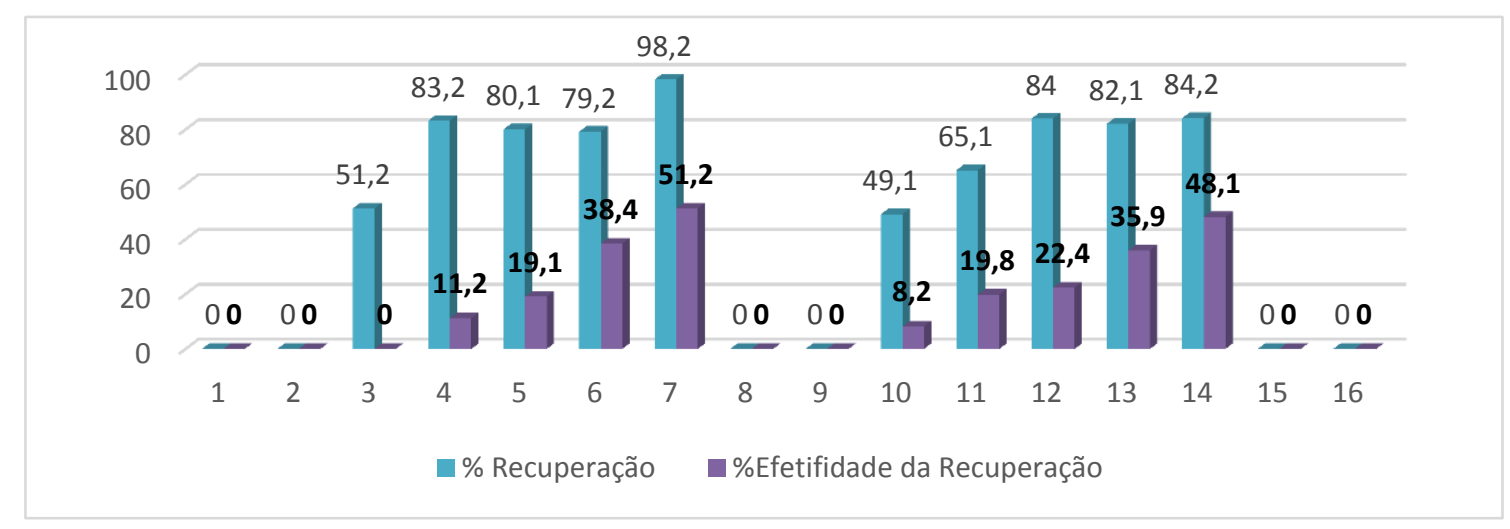

Figura 6 - Resultado de testes preliminares na Recuperação de Casos do Sistema SAA

Como se pode notar nos resultados preliminares obtidos, a taxa de efetividade ainda é baixa, pois a base de casos ainda é pequena. Estudos demonstram que o crescimento da base de conhecimento (casos) leva a um aumento na efetividade do processo de recuperação. Mesmo assim, mais de $25 \%$ dos estudantes com dúvida, 
VI Congresso Brasileiro de Informática na Educação (CBIE 2017)

Anais dos Workshops do VI Congresso Brasileiro de Informática na Educação (WCBIE 2017)

tiveram uma orientação adequada, sem a necessidade de uma intervenção direta do professor. Há de se ressaltar que os momentos com resultados iguais a zero correspondem a mudança de conceito (aula).

Ainda há muito que se testar e aperfeiçoar no sistema SAA. Mas tudo indica que tal sistema pode compor um ferramental importante para os docentes, reduzindo uma das barreiras da utilização das modalidades a distância e híbrida de forma mais extensiva, não só em Algoritmos, mas também em outras disciplinas ou áreas.

\section{Referências}

Alice (2015). What is Alice?. Disponível em: <http://www.alice.org/>. Acesso em: 3 jul.

Azul, A. A. E Mendes, A. J. (2015). EDDL: Um Programa Didático sobre Estruturas de Dados Dinâmicas Lineares. $3^{\mathrm{o}}$ Simpósio Investigação e Desenvolvimento de Software Educativo - 1998. Évora, Portugal. Disponível em: http://www.minerva.uevora.pt/simposio/comunicacoes/Mendes/Eddl.html. Acesso: 15 jun.

Black, P. E. (2015). Dictionary of Algorithms and Data Structures. NIST (National Institute of Standards and Technology). Disponível em http://xlinux.nist.gov/dads/. Acesso: 02 jul.

Borges, M. A. F.(2002). Avaliação de uma Metodologia Alternativa para a Aprendizagem de Programação. VIII Workshop de Educação em Computação - WEI 2000. Curitiba, PR.

Cares, P. L. L.(2002). Ambiente para teste de mesa utilizando fluxograma. Trabalho de Conclusão (Graduação)-Faculdade de Ciência da Computação, Universidade do Vale do Itajaí, Itajaí.

Clark, T. "Attitudes of higher education faculty toward distance education: A national survey". American Journal of Distance Education, Published online: 24 Sep 2009. Disponível em: $<$ http://dx.doi.org/10.1080/08923649309526820>. Acessado em 2804-2017.

Delgado, C., Xexeo, J. A., Souza, I. F., Rapkiewicz, C. E., Pereira Jr, J. (2005). Identificando competências associadas ao aprendizado de leitura e construção de algoritmos. In: XIII Workshop sobre Educação em Computação. São Leopoldo, RS.

Dellazzana, F.(2015). TED - Tutorial de Estruturas de Dados. Universidade do Vale do Itajaí - 2004. Disponível em http://www.tutorialdeestruturadedados.8m.com. Acesso: 02 jul.

Grace, Lauris J. and Smith, Peter J. (2001) "Flexible delivery in the Australian vocational education and training sector: Barriers to success identified in case studies of four adult learners". Distance Education, 22(2), 196-211.

Guri-Rosenblit, S. "Distance education and e-learning: not the same thing. Higher Education. June 2005, V. 49, Issue 4, pp 467-493. doi:10.1007/s10734-004-0040-0. Acessado em 28-04-2017. 
VI Congresso Brasileiro de Informática na Educação (CBIE 2017)

Anais dos Workshops do VI Congresso Brasileiro de Informática na Educação (WCBIE 2017)

Hare, C. and McCartan, A. (1996) "Maximising resources in search of quality: Identifying factors to enable thee integrative uso of IT in teaching and learning." Innovations in Education and Training International, 33(4), 178-184.

Kolodner, J. (1993). “Case-Based Reasoning”, 612 pages, Morgan Kaufman Publishers.

Maguire, L.L. "Literature Review - Faculty Participation in Online Distance Education: Barriers and Motivators. Publicado em 12/3/2008. Disponível em $<$ http://www.bu.edu/ssw/files/pdf/Literature-Review-Faculty-Participation-inOnline-Distance-Education_-Barr2.pdf>. Acessado em 28-04-2017.

Metcalf, T. (1997). "Distance education: the issue of faculty time". 5th Annual Distance Education Conference: 1997 Conference Proceedings. Texas A\&M, Center for Distance Education Research.

Muilenburg, L. and Berge, Z.L. "Barriers to distance education: A factor-analytic stydy", American Journal of Distance Education, Published online: 24 Sep 2009. http://dx.doi.org/10.1080/08923640109527081 Acessado em 28-04-2017.

Pajo, Karl and Wallace, Catherine (1999). "Barriers to the uptake of web-based technology by university teachers". Journal of Distance Education, 16(1), 70-84.

Pereira Júnior, J. C. R.; Rapkiewicz, C. E. (2004). O Processo de Ensino e Aprendizagem de Algoritmos e Programação: Uma Visão Crítica da Literatura. In: III Workshop de Educação em Computação e Informática do Estado de Minas Gerais, WEIMIG'04, Belo Horizonte - MG.

Piva Jr, D.; Nakamiti, G.; Engelbrecht, A.; Bianchi, F. (2012). Algoritmos e Programação de Computadores. Rio de Janeiro:Campus/Elsevier, 2012.

Piva Jr., D.; Cortelazzo, A. L. ; Freitas, F.A. ; Belo, R.O. "Sistema de Avaliação da Aprendizagem (SAA): Operacionalização da Metodologia Flipped Classroom". In: 22o. Congresso Internacional ABED Educação a Distância, 2016, Águas de Lindóia. Anais do 22o CIAED. São Paulo, SP: ABED, 2016.

Priesnitz Filho, W.; Abegg, I.; Simonetto, E.O. (2012). Uma abordagem diferenciada no ensino de algoritmos através da utilização de uma lousa digital. Revista GEINTEC, ISSN 2237-0722, São Cristovão, SE, vol 2, n. 2, p. 129-137.

Rodrigues Jr, M.C. (2004). Experiências Positivas para o Ensino de Algoritmos. Disponível em http:/www.uefs.br/erbase2004/documentos/weibase/ Weibase2004Artigo001.pdf (acessado em 02/jul/2015).

Santos, C.C.; Almeida, T.I.J.P.; Matayoshi, W.P.; Orbato, D.R.S. (2014). “Análise de ferramentas para ensino de algoritmo". $8^{\circ}$. EnTec - Encontro de Tecnologia da UNIUBE: Uberaba/MG, http://www.uniube.br/entec, 28 a 30 de outubro.

Scratch. (2015). Sobre o Scratch (documentação do Scratch no site). Disponível em $<$ https://scratch.mit.edu/about/>. Acesso em: 03 de jul.

Tarouco, L. (2005). Jogos educativos via WWW, Disponível em: http://penta3.ufrgs.br/animacoes/JogosEducacionais/ Acesso: 25 de nov 2005.

Watson, I. (1997) "Applying Case-Based Reasoning: techniques for enterprise systems". San Francisco: Morgan Kaufmann, 1997. 